\title{
ÉTAT ACTUEL DES REBOISEMENTS RÉALISÉS PENDANT LA PÉRIODE 1935-1939 DANS LES ZONES INCENDIÉES DE LA FORÊT DE FONTAINEBLEAU
}

\author{
C. JACQUIOT
}

Class. Oxford $434(44 \times$ REGION PARISIENNE)

La forêt de Fontainebleau est occupée sur presque le tiers de sa surface par des terrains pauvres et arides, souvent accidentés : sables et grès silicieux ou dépôts calcaires des vallées sèches impropres à la formation de peuplements complets d'essences feuillues spontanées (chênes rouvre, pédonculé et pubescent, hêtre et accessoirement charme). La végétation spontanée des terrains silicieux est une lande à éricacées parsemée de genévriers et de bouleaux : bouleau verruqueux en général, bouleau pubescent sur les " platières » où la table de grès, à fleur de sol, retient l'eau en formant des mares permanentes ou temporaires. Dès le XVIle siècle les forestiers cherchèrent à boiser ces " déserts " en introduisant des essences moins exigeantes que les feuillus existants. Les boisements de Pin maritime tentés au XVII e siècle furent décimés par l'hiver de 1709 et après les nombreux essais infructueux de plantation d'essences feuillues qui se poursuivirent pendant la plus grande partie du XVIII ${ }^{e}$ siècle, l'inventaire de 1791 faisait encore état de 7900 arpents (4000 ha environ) de vides. C'est seulement à partir de cette époque que l'introduction du Pin sylvestre, à l'initiative de Lemonnier, apporta au problème une solution satisfaisante, tout au moins sur le plan de la production ligneuse. Les forestiers du XIXe siècle poursuivirent leurs efforts dans cette voie et dans un article publié en 1861 dans les Annales forestières, Marrier de Boisdhyver soulignait que l'introduction des pins, essentiellement le Pin sylvestre et accessoirement le Pin laricio de Corse, "essences dont la rusticité a pu seule triompher de l'ingrate nature du sol ", avait permis le boisement de 4600 ha de " déserts».

Le succès était d'autant plus complet qu'il s'est révélé que le Pin sylvestre, ainsi d'ailleurs que le Pin maritime et le Pin laricio de Corse, se régénèrent naturellement à Fontainebleau. Malheureusement les peuplements de pins, en raison de leur couvert léger, laissent subsister une végétation de fougère aigle et d'éricacées qui permet la propagation du feu. En outre la podzolisation liée aux pins comporte la formation d'une couche épaisse de débris végétaux où le feu peut couver pendant plusieurs jours.

L'histoire de la forêt de Fontainebleau est ainsi marquée par la répétition de sinistres très graves à chaque période de sécheresse. Après les incendies, la régénération naturelle des pins, le plus souvent quelques années après celle des bouleaux, rétablit la végétation forestière, mais dans une forêt aussi fréquentée cette destruction répétée de peuplements précieux, même s'ils se reconstituent naturellement, ne peut être aisément acceptée et incite à rechercher d'autres solutions. 


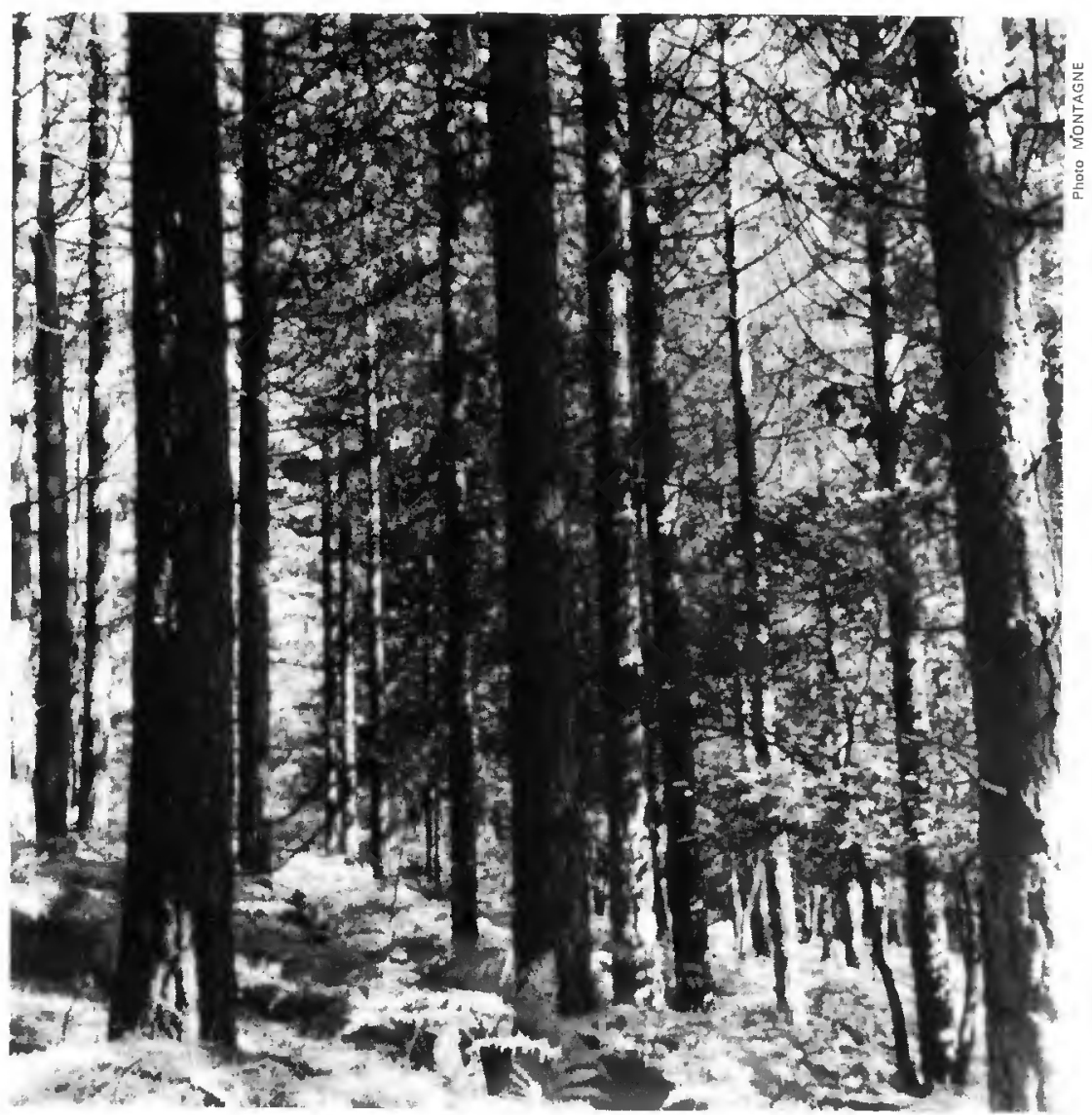

Canton de Rocher Boulin - Peuplement de Douglas et Épicéa mélangés

En 1935, il avait été créé un fonds de chômage, sur lequel des crédits avaient été attribués aux Eaux et Forêts. A la suite des incendies des années précédentes, notamment de l'année 1933, il existait alors en forêt de Fontainebleau environ 500 ha de vides qui, en l'absence d'intervention se seraient spontanément reboisés en bouleaux et pin sylvestre. Le bon sens incitait à utiliser les moyens mis à notre disposition pour chercher à établir dans ces vides des peuplements moins vulnérables aux incendies que la pineraie, en faisant appel à des essences à couvert assez dense pour éliminer la végétation d'éricacées et de fougères. C'est dans cet esprit que j'élaborai le plan des travaux de reboisements ( ${ }^{1}$ ) exécuté en partie de 1935 à 1939 et dont la poursuite fut interrompue par la guerre. En principe ce plan prévoyait l'introduction d'essences diverses. Pour l'Épicéa commun des essais antérieurs sur des surfaces plus ou moins importantes avaient montré qu'il pouvait donner des résultats acceptables mais ne se régénérait que sporadiquement. Pour d'autres essences qui n'avaient été introduites précédemment que par pieds isolés ou petits groupes, comme le Douglas, le Sapin de Nordmann ou l'Épicéa d'Orient, et dont la croissance s'était montrée très satisfaisante, le plan de reboisement gardait un caractère expérimental avec cependant une présomption de réussite.

(1) Jacquiot (C.) - - Note sur les problèmes écologiques et les problèmes pratiques de boisement artificiel des vides et de substitution d'essences en forêt de Fontainebleau. Travaux des naturalistes de la vallée du Loing, fasc. 10, 1938, pp. 5-18. 
Pour éviter les inconvénients des peuplements purs de conifères, il était prévu de mélanger en proportions égales une essence résineuse et une essence feuillue : Chêne rouge d'Amérique et Châtaignier en terrain silicieux ou Hêtre en terrain calcaire. Dans ce dernier cas l'introduction du Hêtre ne devait intervenir que trois ou quatre ans après l'installation du résineux, capable alors de fournir un abri aux jeunes hêtres plantés en interlignes. Les plants utilisés dans les plantations en plein étaient des plants de trois ans repiqués mais, pour constituer rapidement des pare-feu, des hautes-tiges principalement d'Épicéa ou de Douglas furent plantées en double ligne le long des allées forestières dans les zones les plus menacées. Suivant le même principe, des plantations de hautes-tiges de Douglas, de Cyprès de Lawson ou de Thuya géant furent installées le long de la voie ferrée où circulaient alors des trains à vapeur, source importante d'incendies. La S.N.C.F. contribua d'ailleurs à cette partie du programme.

A la fin de l'hiver 1938-1939, les plantations réalisées couvraient environ 200 ha. Dès les premières années deux causes d'échec étaient apparues. En premier lieu les dégâts du lapin : bien que la population de ce rongeur fût peu abondante, l'attirance exercée par les plants introduits artificiellement entraîna dans certains cantons des dommages très importants. Si dans les parcelles les poins atteintes les dégâts subis par l'Épicéa et surtout par le Douglas furent négligeables, le Chêne rouge fut pratiquemont éliminé. Le Châtaignier fut beaucoup moins éprouvé. Les dégâts des grands animaux, qui s'étaient manifestés au début, furent arrêtés par la pose de quelques rangs de fils barbelés. Après quelques années ces clôtures purent être enlevées sans que les dégâts se renouvellent. En second lieu, sur les versants exposés au midi la plupart des jeunes plants de Douglas se desséchèrent au cours du printemps de 1938, où les conditions météorologiques avaient été très comparablos à celles du printemps $1976\left(^{2}\right)$. Cos versants furent alors reboisés en Pin laricio de Corse associé au Châtaignier. Par contre sur les versants nord et dans les fonds des vallées, la reprise fut presque totale. Des incendies accidentels localisés détruisirent au total une vingtaine d'hectares environ de plantations. Par contre, l'incendie criminel allumé par l'ennemi en 1943 pour chasser les maquis opérant dans le massif des Trois-Pignons, et qui parcourut plus de 1700 ha dont 700 ha en forêt domaniale, anéantit 70 ha de plantations dans les cantons ouest (Aiguisoires, gorge aux Archers, Bois-Rond).

En définitive, les peuplements obtenus par ces travaux de reboisement couvrent environ 95 ha, dont la plus grandc partie en terrain non calcaire (sables et grès au rocher Boulin, au rocher Bouligny, au rocher Cassepot, graviers des terrasses alluviales à la Queue de Fontaine et au Bois-la-Dame). Ces peuplements d'Épicéa ou de Douglas, âgés d'une quarantaine d'années, sont complets et fermés et le but recherché de l'élimination de la flore combustible a été atteint. Les doubles rangées de plantations de hautes-tiges, en particulier, ont formé des bandes pare-feu efficaces de part et d'autre des chemins. Elles ont de plus une valeur esthétique certaine, comme on peut notamment le voir au rocher Boulin et dans les gorges de Franchard.

Un autre point important est la croissance très satisfaisante du Douglas. On peut noter aussi que des semis naturels apparaissent déjà dans leur voisinage. Cette essence doit donc prendre une place importante dans les sables et grès. Sur les versants exposés au sud son introduction artificielle ne peut réussir en plein découvert, comme l'a montré l'expérience de 1938, mais elle est possible dans des trouées peu étendues ou sous l'abri d'un peuplement de bouleaux ou de pins. Et une fois installés, le Douglas peut supporter des périodes de sécheresse comme l'a montré l'excellente tenue des peuplements pendant les périodes de sécheresse survenues depuis 1939 et tout particulièrement pendant celle de 1976. II permettra d'obtenir au moins un compartimentage des zones exposées aux incendies. De plus sa croissance satisfaisante et les qualités de son bois offrent un intérêt économique certain, s'ajoutant aux avantages esthétiques et au rôle de protection déjà évoqués.

(2) Rappelons que le total des prècipitations pour la période 1er mars-30 juin fut de $84 \mathrm{~mm}$ en 1938 et $65 \mathrm{~mm}$ en 1976 . La moyenne est $212 \mathrm{~mm}$. 
D'autres essences n'ont pu faire l'objet que d'essais très limités en raison des faibles ressources des pépiniéristes. Parmi les sapins, il s'est confirmé que le Sapin de Nordmann méritait de prendre place dans les reboisements ainsi que le Sapin de Céphalonie. Il en est de même de l'Épicéa d'Orient.

Les boisements réalisés pendant la période 1935-1939 ont permis, malgré les destructions de la guerre, d'installer dans des parcelles incendiées des peuplements moins vulnérables que les pineraies. Ils ont aussi apporté des indications précieuses sur les possibilités offertes par diverses essences pour le reboisement des zones incendiées à Fontainebleau et ont eu enfin une incidence esthétique heureuse en créant dans des cantons dévastés un nouveau type de paysage forestier.

Clément JACQUIOT

Ingénieur en chef du G.R.E.F. LABORATOIRE DE BIOLOGIE VÉGÉTALE

Route de la Tour-Denécourt 77300 FONTAINEBLEAU

\section{PINS MARITIMES EXCEPTIONNELS}

\section{au lieut-dit "LES SOURCES de GAZINET», Commune de CESTAS}

A quelques dizaines de mètres du restaurant «Les Sources " un remarquable site naturel comprenant des pins de 120 ans, des chênes de 300 ans, un sous-bois touffu et des sources d'eau ferrugineuses d'un débit considérable, mérite vraiment quelques instants d'intérêt.

Des mesures effectuées sur les plus beaux pins témoignent du caractère exceptionnel des sujets testés.

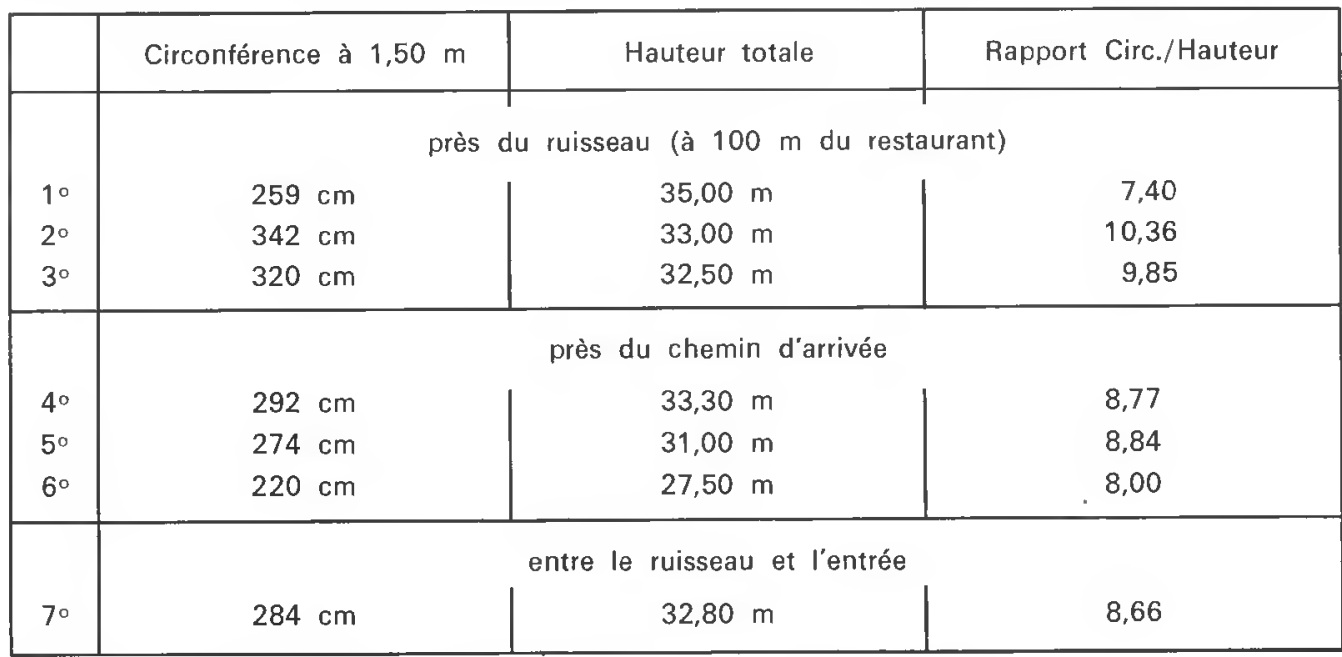

D'autre part trois chablis sont au contact du sol et mesurent de 30 à 33 mètres.

Laboratoire de Sylviculture et Écologie de la Pinède Landaise (I.N.R.A.) 